\title{
Coaxial Biopsy
}

National Cancer Institute

\section{Source}

National Cancer Institute. Coaxial Biopsy. NCI Thesaurus. Code C159942.

A biopsy system that uses a special needle to permit obtaining multiple samples through a single puncture. 